\title{
Magnetization processes in single domain elliptical permalloy thin films
}

\author{
Zung-Hang Wei ${ }^{\mathrm{a}}$, Mei-Feng Lai ${ }^{\mathrm{a}, *}$, Ching-Ray Chang ${ }^{\mathrm{a}}$, N.A. Usov ${ }^{\mathrm{b}}$, \\ J.C. $\mathrm{Wu}^{\mathrm{c}}$, Jun-Yang Lai ${ }^{\mathrm{a}}$ \\ ${ }^{a}$ Department of Physics, National Taiwan University, Taipei, 10617, Taiwan \\ ${ }^{\mathrm{b}}$ Troitsk Institute for Innovation and Fusion Research, Troitsk, Moscow region, 142190, Russia \\ ${ }^{\mathrm{c}}$ Department of Physics, National Changhua University of Education, Changhua, 500, Taiwan
}

\begin{abstract}
We use the micromagnetic simulation to investigate the reversible and irreversible magnetization processes of the single-domain elliptical permalloy thin films. The reversible magnetization curve without hysteresis obtained by magnetizing the single-domain elliptical permalloy thin film along the hard axis is almost linear as are single-domain ellipsoidal particles. For irreversible processes with hysteresis, the switching field of the ellliptical thin film is reduced compared with that predicted by the Stoner-Wohlfarth model due to the occurrence of non-uniform reversal.
\end{abstract}

(C) 2004 Elsevier B.V. All rights reserved.

PACS: $75.60 . \mathrm{Jk} ; 75.75 .+\mathrm{a}$

Keywords: Stoner-Wohlfarth model; Micromagnetic simulation; Magnetization reversal

Various spintronic devices such as magnetic random access memory (MRAM) use single-domain magnetic thin films as unit cells. A bit can be written by magnetic fields generated from electric currents passing through two perpendicular electrodes above and below each cell. It is therefore important to understand the magnetization properties in thin film elements in order to obtain the uniform performance and repeatability during accessing the unit cell. In this article we investigate the magnetization curves of the reversible processes by magnetizing the elements along the hard axis direction, and the hysteresis loops of the irreversible processes by remagnetizing the elements along the easy axis direction. Hysteresis loops corresponding to various field directions are also studied.

In our simulation, we use the Landau-LifshitzGilbert [1] equation to investigate the ferromagnetic permalloy thin film. The typical parameters of the Permalloy material are as follows: the exchange constant

\footnotetext{
*Corresponding author. Tel.: +886-2-33665183; fax: +8862-23639984.

E-mail address: d90222026@ms90.ntu.edu.tw (M.-F. Lai).
}

$C=2 A=2 \times 10^{-6} \mathrm{erg} / \mathrm{cm}$, the saturation magnetization $M_{\mathrm{s}}=800 \mathrm{emu} / \mathrm{cm}^{3}$, and the uniaxial anisotropy constant $K_{1}=10^{3} \mathrm{erg} / \mathrm{cm}^{3}$. The particle is divided into an array of small cubic cells with side length $7.5 \mathrm{~nm}$, which is small with respect to the permalloy exchange length $17.7 \mathrm{~nm}$. The equilibrium state is reached when the deviation of the magnetization is smaller than $10^{-8}$ between two successive iterations.

In order to understand the behaviors of the singledomain elliptical thin film under external magnetic field it is helpful to compare the magnetization processes of the ellipse and its corresponding Stoner-Wohlfarth [2] ellipsoid. The demagnetization factors of the corresponding ellipsoid, which has the same volume as the elliptical thin film, can be determined from the BrownMorrish theorem [3]. The magnetization curves of the single-domain ellipsoidal particles are linear [3] when an external field is applied along the hard axis as shown in Fig. 1. For single-domain elliptical elements with different sizes the magnetization curves are close to the corresponding ones of the ellipsoidal particles, but the magnetization rotation towards the external field direction is not a rotation in unison because the 


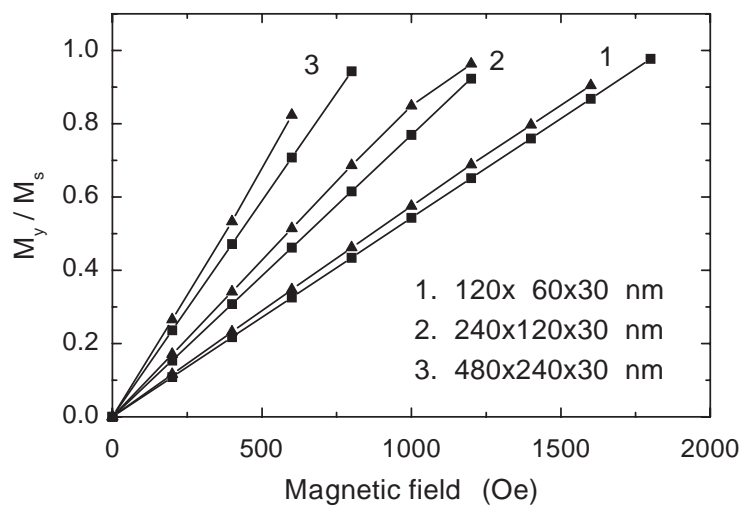

Fig. 1. Magnetization curves of the single-domain elliptical $(\mathbf{\Delta})$ thin films and the corresponding Stoner-Wohlfarth ellipsoidal (ם) particles, which respectively have the same volumes as the three elliptical thin films, when a field is applied along the hard axis. Three sets of curves correspond to three different particles with dimensions indicated by long-axis $\times$ short-axis $\times$ thickness.

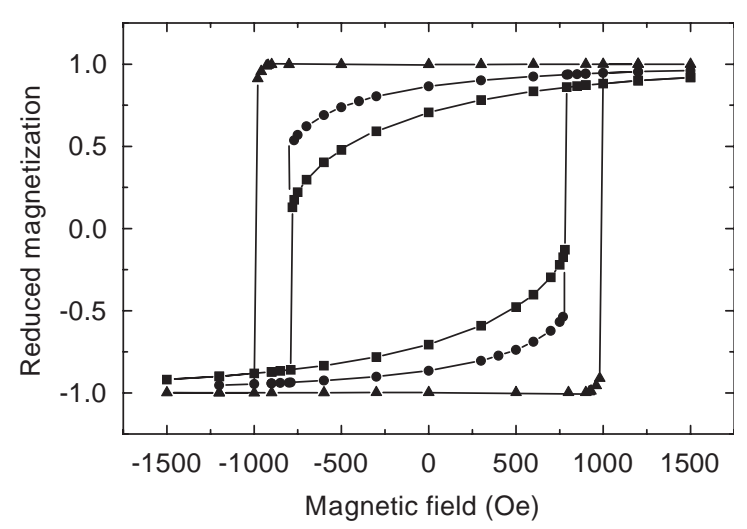

Fig. 2. Hysteresis loops of the permalloy elliptical thin film at various field angles with respect to the particle's long axis. The angles are $0^{\circ}(\boldsymbol{\Delta}), 30^{\circ}(\bullet)$, and $45^{\circ}(\boldsymbol{\square})$. The dimension of the particle is $360 \mathrm{~nm}$ in long axis, $120 \mathrm{~nm}$ in short axis, and $30 \mathrm{~nm}$ in thickness.

demagnetization field inside is not uniform. In contrast to the Stoner-Wohlfarth ellipsoid, which saturates under a critical field, the elliptical elements do not saturate under a finite field but the magnetization only rotates gradually as the field increases. This magnetization process is reversible and hence the hysteresis is zero.

For the irreversible cases, we simulate the hysteresis loops of elliptical elements with different sizes and different field angles with respect to the element's long axis as shown in Fig. 2. The hysteresis loop of elliptical elements is approximately rectangular when an external field is along the particle's long axis, but the corner is rounded compared with the Stoner-Wohlfarth ellipsoidal particle because the magnetization becomes non-
Table 1

Switching fields of elliptical particles $\left(H_{\mathrm{c}}\right)$ and those of the corresponding Stoner-Wohlfarth ellipsoids $\left(H_{\mathrm{c}, \mathrm{SW}}\right)$ with different sizes and aspect ratios

\begin{tabular}{llrc}
\hline Size $(\mathrm{nm})$ & Field angle $($ deg. $)$ & $H_{\mathrm{c}, \mathrm{SW}}(\mathrm{Oe})$ & $H_{\mathrm{c}}(\mathrm{Oe})$ \\
\hline $360 \times 120 \times 30$ & 0 & 1787.20 & 1000 \\
& 30 & 937.85 & 790 \\
$720 \times 240 \times 30$ & 0 & 894.87 & 790 \\
& 35 & 1149.00 & 520 \\
& 45 & 603.46 & 440 \\
$240 \times 120 \times 30$ & 0 & 575.80 & 440 \\
& 30 & 1300.00 & 750 \\
& 45 & 682.53 & 495 \\
& & 651.25 & 470 \\
\hline
\end{tabular}

uniform before switching. Besides, the switching field reduces significantly compared to the Stoner-Wohlfarth ellipsoidal particle due to the non-uniform remagnetization process. This process initiates with several vortices nucleated at the boundary, followed by the penetrating of these vortices across the element, and then completes by the annihilation of the vortices. When the external fields are applied in different directions, the hysteresis loops show similar behaviors as the Stoner-Wohlfarth ellipsoidal particle and the switching fields are also reduced.

Table 1 shows the simulation results of the switching fields of elliptical elements with different sizes and the corresponding analytical results of the Stoner-Wohlfarth model. For the Stoner-Wohlfarth model the switching fields are reduced when the fields are not along the easy axis. It is the same for the elliptical elements. For the two elliptical elements with aspect ratio $3: 1$, the switching fields are almost the same for $30^{\circ}$ and $45^{\circ}$ field directions because before reversal the total magnetic moment deviates from the easy axis towards the field direction, and the magnetization at the two ends of the long axis is bended by the boundary. Vortices are easily nucleated in the regions where the magnetization curvature is large enough. However, for the elliptical elements with aspect ratio 2:1, the curvature at the two ends is smaller so vortices are not easily nucleated as the above case, and therefore the field direction has more apparent influence on the switching fields.

\section{References}

[1] M.E. Schabes, J. Magn. Magn. Mater. 95 (1991) 249.

[2] E.S. Stoner, et al., Philos. Trans. R. Soc. London Ser. A 240 (1948) 599.

[3] W.F. Brown Jr., Micromagnetics, Interscience, New York, 1963. 